\title{
New Bound States of Heavy Quarks at LHC and Tevatron
}

\author{
C.R. Das ${ }^{1 *}$, C.D. Froggatt ${ }^{2 \dagger}$, L.V. Laperashvili ${ }^{\ddagger}$ and H.B. Nielsen ${ }^{4} \S$ \\ 1 Centre for Theoretical Particle Physics, \\ Technical University of Lisbon, Lisbon, Portugal \\ ${ }^{2}$ Department of Physics and Astronomy, \\ Glasgow University, Glasgow, Scotland \\ ${ }^{3}$ Institute of Theoretical and Experimental Physics, \\ Moscow, Russia \\ 4 The Niels Bohr Institute, Copenhagen, Denmark
}

\begin{abstract}
The present paper is based on the assumption that heavy quarks bound states exist in the Standard Model (SM). Considering New Bound States (NBS) of topanti-top quarks (named T-balls) we have shown that: 1) there exists the scalar $1 S$-bound state of $6 t+6 \bar{t} ; 2)$ the forces which bind the top-quarks are very strong and almost completely compensate the mass of the twelve top-anti-top-quarks in the scalar NBS; 3) such strong forces are produced by the Higgs-top-quarks interaction with a large value of the top-quark Yukawa coupling constant $g_{t} \simeq 1$. Theory also predicts the existence of the NBS $6 t+5 \bar{t}$, which is a color triplet and a fermion similar to the $t^{\prime}$-quark of the fourth generation. We have also considered the "b-quarkreplaced" NBS, estimated the masses of the lightest fermionic NBS: $M_{N B S} \gtrsim 300$ $\mathrm{GeV}$, and discussed the larger masses of T-balls. We have developed a theory of the scalar T-ball's condensate and predicted the existence of three SM phases. Searching for heavy quark bound states at the Tevatron and LHC is discussed. We have constructed the possible form-factors of T-balls, and estimated the charge multiplicity coming from the T-ball's decays.
\end{abstract}

*crdas@cftp.ist.utl.pt

${ }^{\dagger}$ c.froggatt@physics.gla.ac.uk

†laper@itep.ru

$\S$ hbech@nbi.dk 


\section{Introduction}

Although the Standard Model (SM) was confirmed by all experiments of the world accelerators, the mechanism of the Electroweak (EW) symmetry breaking (EWSB) has not yet been tested. According to the SM, the Higgs boson is responsible for generating the masses of fermions due to the Higgs mechanism. However, the mass of the Higgs boson is not predicted by theory.

Direct searches in the previous experiments (mainly at LEP2 [1]) set a lowest limit for the Higgs boson mass $M_{H}$ :

$$
M_{H} \gtrsim 114.4 \mathrm{GeV} \text { at } 95 \% \mathrm{CL} \text {. }
$$

The recent Tevatron result [2] is:

$$
114 \lesssim M_{H} \lesssim 158 \mathrm{GeV}
$$

at $97 \%$ C.L. if direct limit of $114 \mathrm{GeV}$ from LEP is excluded in thw fit. We hope that LHC will provide a solution of main puzzles of EWSB.

The Higgs boson couples more strongly to the heavy top quarks than to the light ones. As a result, the Higgs exchanges between top quarks produce new type of bound states [3 16].

The present paper is devoted to the properties of the new bound states (NBS): estimates of their masses and observation at modern colliders (Tevatron, LHC, etc.). The predictions of Refs. [3-12] are:

- There exists a scalar $1 S$-bound state of $6 t+6 \bar{t}$. The forces which bind these topquarks are so strong that almost completely compensate the mass of the 12 top-quarks forming this bound state.

- There exists a new bound state $6 t+5 \bar{t}$, which is a fermion similar to the quark of the fourth generation having quantum numbers of top quark.

- Theory also predicts the existence of new bound states with b-quark replaced the t-quark: for example, NBS $n_{b} b+\left(6 t+6 \bar{t}-n_{b} t\right)$, etc., where $n_{b}=1, \ldots 6$.

A new (earlier unknown) bound state $6 t+6 \bar{t}$, which is a color singlet (that is, 'white' state), was first suggested by Froggatt and Nielsen in Ref. [5]. Now all these NBS are named T-balls, or T-fireballs.

\section{Higgs and gluon interactions of quarks}

If the Higgs particle exists, then between quarks $q q$, quarks and anti-quarks $q \bar{q}$, and also between anti-quarks $\bar{q} \bar{q}$ there exist virtual exchanges by Higgs bosons (see Fig. 1), leading only to the attractive forces.

It is well-known that the bound state $t \bar{t}$ - so called toponium - is obliged to the gluon virtual exchanges of Fig. 2. Among a considerable quantity of articles devoted to the toponium, we distinguish the following backward papers [17 22$]$. 


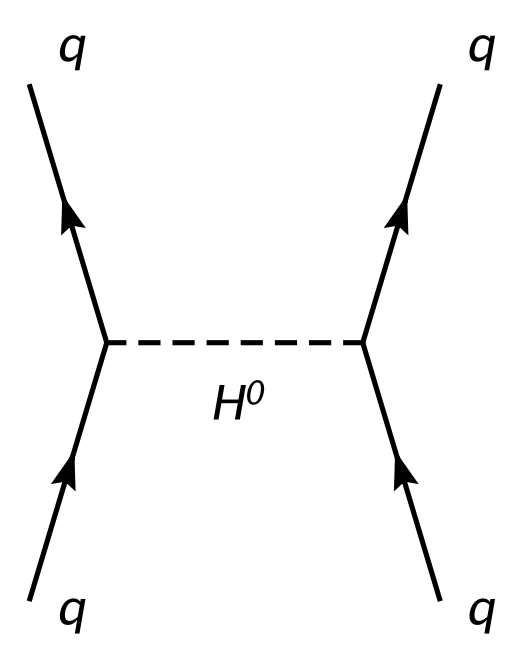

(a)

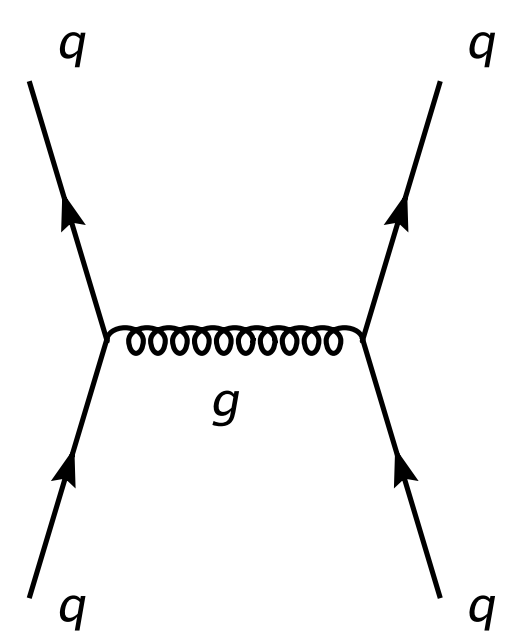

(a)

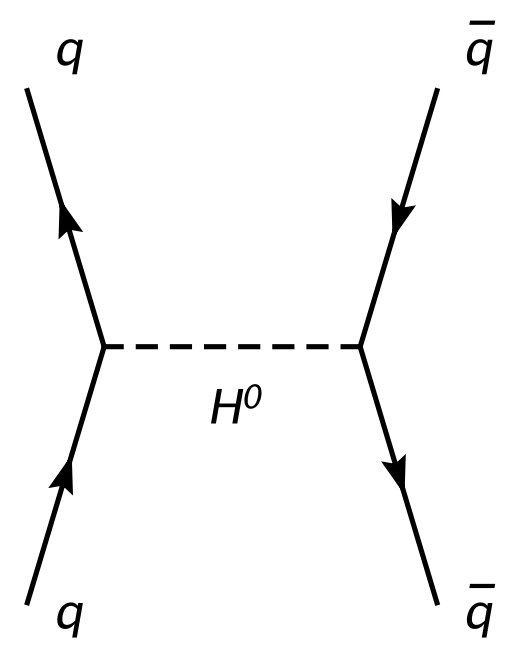

(b)

Fig. 1:

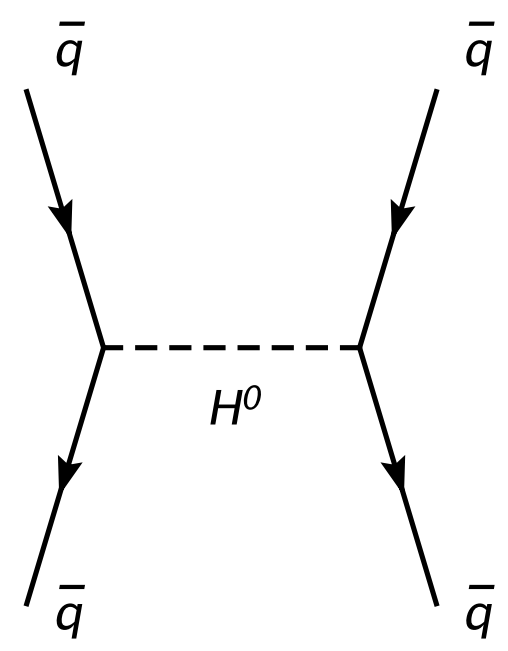

(c)

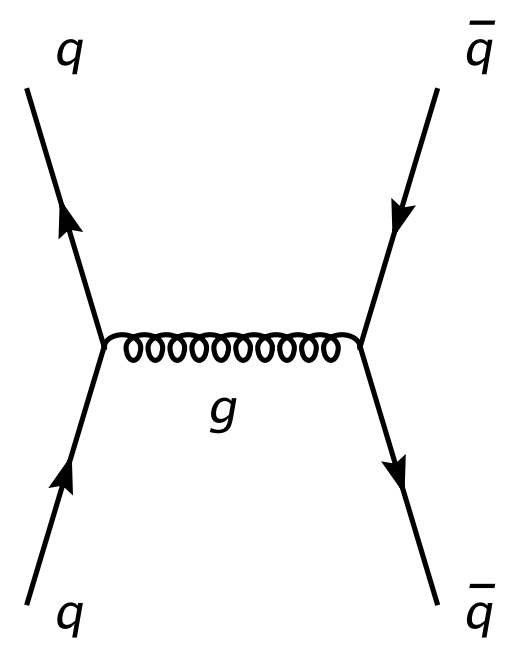

(b)

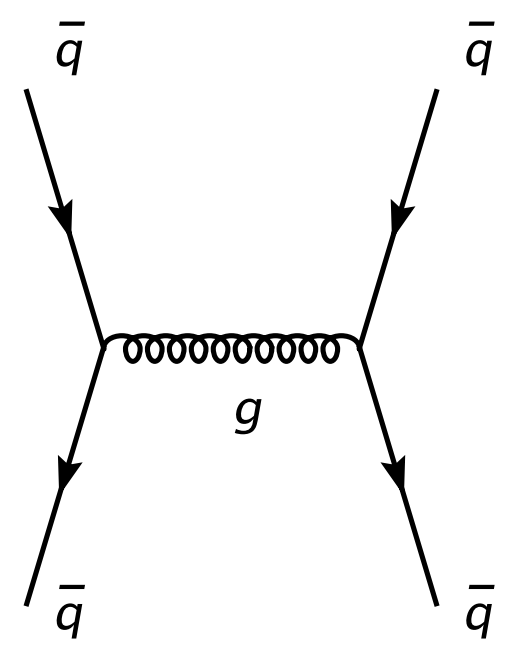

(c)

Fig. 2: 
In the case of the toponium the contributions of the Higgs scalar particles are essential, but less than gluon interactions. Toponium is very unstable due to the decay of the top quark itself. However, putting more and more top and anti-top quarks together in the lowest energy bound states, we notice that the attractive Higgs forces continue to increase. Simultaneously gluon (attractive and repulsive) forces first begin to compensate themselves, but then begin to decrease relatively to the Higgs effect with growth of the number of top-anti-top constituents in the NBS.

The maximum of the binding energy value corresponds to the $1 S$-wave state of the NBS $6 t+6 \bar{t}$. The explanation is simple: top-quark has two spin states and three states of colors: $2 \times 3=6$ degrees of freedom. This means that, according to the Pauli principle, only 6 pairs of $t \bar{t}$ can simultaneously exist in the 'white' $1 S$-wave state. If we try to add more $t \bar{t}$-pairs, then some of them will turn out to the $2 S$-wave state, and the NBS binding energy will decrease at least 4 times. For P-,D-, etc. wave states the NBS binding energy decreases more and more.

\section{T-ball mass estimate}

The kinetic energy term of the Higgs field and the top-quark Yukawa interaction are given by the following Lagrangian density:

$$
L=\frac{1}{2} D_{\mu} \Phi_{H} D^{\mu} \Phi_{H}+\frac{g_{t}}{\sqrt{2}} \overline{\psi_{t L}} \psi_{t R} \Phi_{H}+\text { h.c. },
$$

where $\Phi_{H}$ and $\psi_{t}$ are the Higgs and top-quark fields, respectively, and $g_{t}$ is the Yukawa coupling constant of their interaction.

The VEV of the Higgs field in the EW-vacuum is:

$$
v=<\left|\Phi_{H}\right|>=246 \mathrm{GeV} .
$$

According to the Salam-Weinberg theory the top-quark mass $M_{t}$ and the Higgs mass $M_{H}$ are given by the following relations:

$$
M_{t}=\frac{g_{t}}{\sqrt{2}} v \quad \text { and } \quad M_{H}^{2}=\lambda v^{2},
$$

where $\lambda$ is the Higgs self interaction coupling constant.

According to Ref. [23],

$$
M_{t} \approx 172.6 \mathrm{GeV} \text {, }
$$

and

$$
g_{t} \approx 0.93
$$

Let us imagine now that the NBS is a bubble in the EW-vacuum and contains $N_{\text {const. }}$. top-like constituents. It is known that insight the bubble (bag) the Higgs field can modify 
its VEV. Implications related with this phenomenon have been discussed in Refs. 6, 22, 24 28]. Then insight T-balls the VEV of the Higgs field is smaller than $v$ :

$$
v_{0}=<\left|\Phi_{h}\right|>, \quad \text { where } \frac{v_{0}}{v}<1,
$$

and the effective masses insight the bubble (bag) are smaller than the corresponding experimental masses:

$$
m_{t, h}=\frac{v_{0}}{v} M_{t, H}
$$

In this case the attraction between two top (or anti-top) quarks is presented by the Yukawa type of potential:

$$
V(r)=-\frac{g_{t}^{2} / 2}{4 \pi r} \exp \left(-m_{h} r\right)
$$

Assuming that the radius $R_{0}$ of the bubble is small:

$$
m_{h} R_{0}<<1
$$

we obtain the Coulomb-like potential:

$$
V(r) \simeq-\frac{g_{t}^{2} / 2}{4 \pi r}
$$

The attraction between any pairs $t t, t \bar{t}, \overline{t t}$ is described by the same potential (12).

By analogy with Bohr Hydrogen-atom-like model, the binding energy of a single topquark relatively to the nucleus containing $Z=N_{\text {const. }}-1$ top-quarks have been estimated in Refs. [5] [7]. The total potential energy for the NBS with $N_{\text {const. }}=12$ is:

$$
V_{t o t}(r)=-11 \frac{g_{t}^{2} / 2}{4 \pi r}
$$

Here we would like to comment that the value of the mass $m_{h}$, which belongs to the Higgs field insight the NBS $6 t+6 \bar{t}$, can just coincide with estimates given by Refs. [13 16]. The results: $\max \left(\mathrm{m}_{\mathrm{h}}\right)=29 \mathrm{Gev}$ and $\max \left(\mathrm{m}_{\mathrm{h}}\right)=49 \mathrm{Gev}$ correspond to Ref. [14] and Ref. [16], respectively.

Considering a set of Feynman diagrams (the Bethe-Salpeter equation) and including the contributions of all ( $\mathrm{s}-, \mathrm{t}-$ and $\mathrm{u}-$ ) channels for the Higgs and gluon exchange forces (see Ref. [6]), we obtain the following Taylor expansion:

$$
M_{T}^{2}=\left(N_{\text {const. }} M_{t}\right)^{2} \times\left\{1-2\left(N_{\text {const. }}-1\right)\left(\frac{N_{\text {const. }}}{12}\right)^{2}\left(\frac{g_{t}^{2}+\frac{1}{6} g_{s}^{2}}{\pi}\right)^{2}+\ldots\right\}
$$

Here the QCD coupling constant $g_{s}$ is given by its fine structure constant value at the EW-scale [23]:

$$
\alpha_{s}\left(M_{Z}\right)=g_{s}^{2}\left(M_{Z}\right) / 4 \pi \approx 0.118
$$


Now the value of the total binding energy for arbitrary $N_{\text {const. }}$ is equal to:

$$
E_{T}=N_{\text {const. }}\left(N_{\text {const. }}-1\right)\left(\frac{N_{\text {const. }}}{12}\right)^{2}\left(\frac{g_{t}^{2}+\frac{1}{6} g_{s}^{2}}{\pi}\right)^{2} m_{t} .
$$

The mass of T-ball containing $N_{\text {const. }}$ top or anti-top quarks is:

$$
M_{T}=N_{\text {const. }} m_{t}-E_{T} \text {. }
$$

Approximately this dependence is described by the following expression:

$$
M_{T}=N_{\text {const. }} m_{t}\left\{1-\left(N_{\text {const. }}-1\right)\left(\frac{N_{\text {const. }}}{12}\right)^{2}\left(\frac{g_{t}^{2}+\frac{1}{6} g_{s}^{2}}{\pi}\right)^{2}\right\} .
$$

Below we shall use the following notations: $T_{s}$-ball is a scalar NBS $6 t+6 \bar{t}$, having the spin $S=0$, and $T_{f}$-ball presents the NBS $6 t+5 \bar{t}$, which is a fermion: $\overline{T_{f}}=5 t+6 \bar{t}$.

Let us consider now the condition:

$$
\frac{11}{\pi^{2}} \cdot\left(g_{t}^{2}+\frac{1}{6} g_{s}^{2}\right)^{2}=1 .
$$

In this case the binding energy $E_{T}$ compensates the NBS mass $12 m_{t}$ so strongly that the mass of the scalar $T_{s}$-ball becomes zero:

$$
M_{T_{s}}=11 m_{t}\left\{1-\frac{11}{\pi^{2}} \cdot\left(g_{t}^{2}+\frac{1}{6} g_{s}^{2}\right)^{2}\right\}=0 .
$$

It is necessary to emphasize that the experimental values given by (7) and (15) [23]:

$$
g_{t}^{2} \simeq 0.86 \text { and } g_{s}^{2} \simeq 1.48
$$

are just very close to this limit.

Fig. 3 shows the dependence of T-ball masses on the number of NBS constituents $N_{\text {const. }}$ In the case when $M_{T_{s}}=0$, we have:

$$
M_{T}=N_{\text {const. }} m_{t}\left\{1-\frac{\left(N_{\text {const. }}-1\right)}{11} \frac{N_{\text {const. }}^{2}}{12^{2}}\right\} \text {. }
$$

We easily see that the light scalar Higgs bosons with mass $m_{h}<M_{H}$ can bind the 12 top-like quarks so strongly that the mass $M_{T_{s}}$ becomes almost zero, and even tachyonic: $M_{T_{s}}^{2}<0$. In the last case we obtain the Bose-Einstein condensate of T-balls a new vacuum at the EW-scale [12]. Previously the condensation of $t \bar{t}$, arising from fourfermion interaction models ( [29 31], etc.), was reviewed in Ref. 32]. We have suggested a new type of condensation of top-quarks via T-balls, what is very important for the solution of the hierarchy problem in the SM [10,11]. 


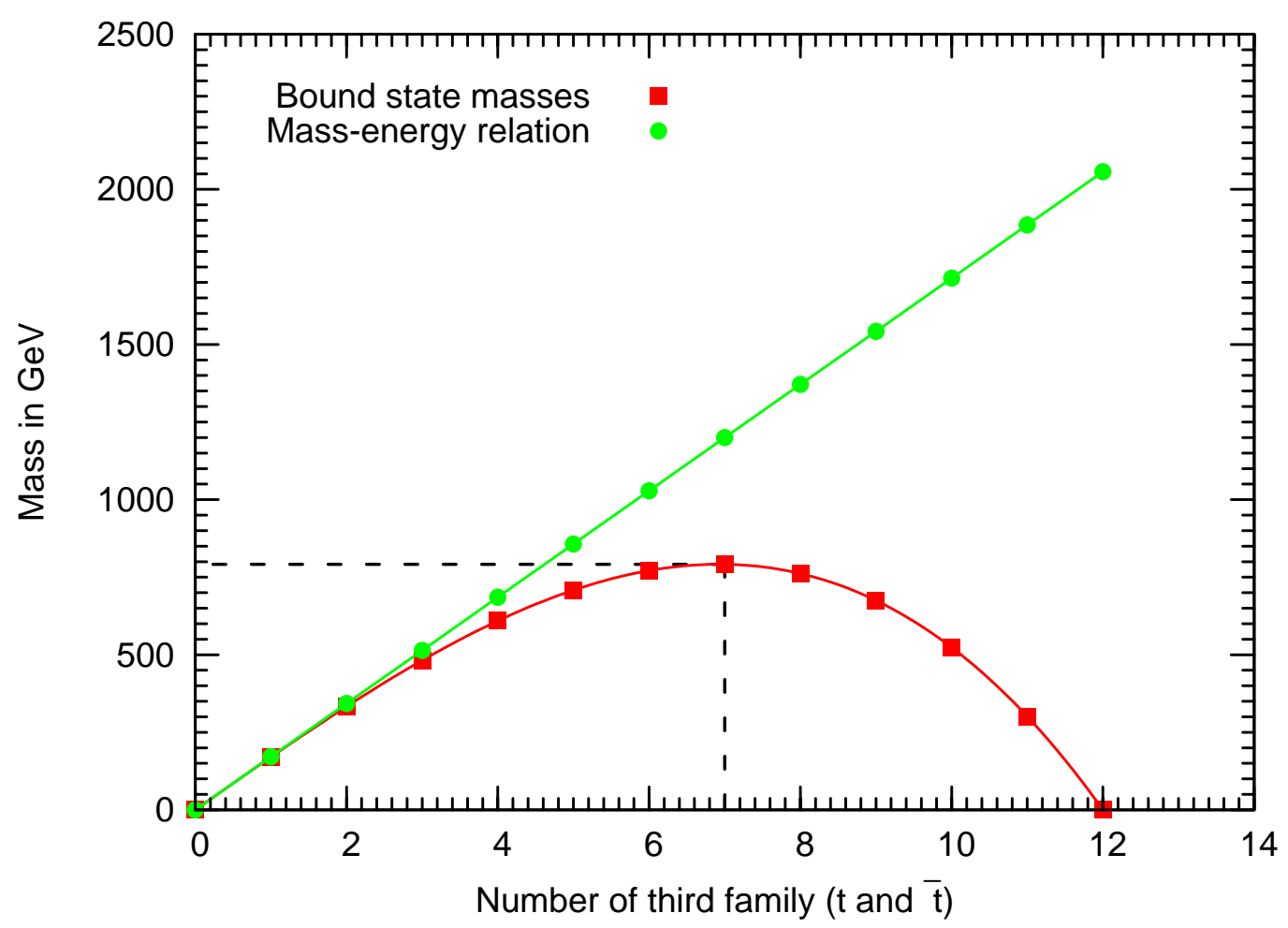

Fig. 3: T-ball mass depending on the number $N_{\text {const. }}$ of the NBS constituents. 


\section{$3.1 \quad \mathrm{~T}_{\mathrm{f}}$-ball mass estimate}

As we have discussed above, the Higgs interaction of the eleven top-anti-top quarks $\left(N_{\text {const. }}=11\right)$ creates a $T_{f}$-ball - a new fermionic bound state $6 t+5 \bar{t}$, which is similar to the $t^{\prime}$-quark of the fourth generation. The estimate of the mass of $T_{f}$-ball $6 t+5 \bar{t}$ by Eq. (22) gives :

$$
M_{T_{f}} \approx 11 m_{t} \cdot 0.236 \gtrsim 300 \mathrm{GeV} .
$$

The detailed analysis of calculation of the NBS-masses was considered in Ref. [7]. We hope that the forthcoming numerical calculations of the T-ball masses by Monte-Carlo simulations on lattice will give us more exact answers.

\section{New "b-replaced" bound states}

Constructing T-balls from $t$ and $\bar{t}$-quarks, we also can take into account considerable contributions of left b-quarks insight NBS [3, 7,,12].

If we had no $b \bar{b}$-pairs in T-balls, then there would be an essential superposition of different states of the weak isospin. The presence of b-quarks in the NBS leads to the dominance of the isospin singlets of EW-interactions only. Now such a "b-replaced" scalar NBS would be stable. We predict the following scalar "b-replaced" NBS:

$$
\begin{aligned}
& T_{s}(b-\text { replaced })=b+5 t+6 \bar{t}, \\
& T_{s}(\bar{b}-\text { replaced })=6 t+\bar{b}+5 \bar{t} .
\end{aligned}
$$

In general case we can construct the following scalar "b-replaced" T-balls:

$$
T_{s}(n b-\text { replaced })=n_{b} b+\left(6 t+6 \bar{t}-n_{b} t\right),
$$

and

$$
T_{s}(n \bar{b}-\text { replaced },)=n_{\bar{b}} \bar{b}+\left(6 t+6 \bar{t}-n_{\bar{b}} \bar{t}\right) .
$$

Of course, we also can construct the fermionic "b-replaced" NBS:

$$
T_{f}(b-\text { replaced })=b+5 t+5 \bar{t},
$$

and

$$
\overline{T_{f}}(\bar{b}-\text { replaced })=5 t+5 \bar{t}+\bar{b} .
$$

In general case we obtain:

$$
T_{f}(n b-\text { replaced })=n_{b} b+\left(6 t+5 \bar{t}-n_{b} t\right),
$$

and

$$
\overline{T_{f}}(n \bar{b}-\text { replaced })=n_{\bar{b}} \bar{b}+\left(5 t+6 \bar{t}-n_{\bar{b}} \bar{t}\right) .
$$


We have $n_{b}, n_{\bar{b}}=1, \ldots 6$ in Eqs. (26) -(31).

There is a simple way to estimate the mass of the "b-replaced" T-ball with one tquark replaced by a b-quark. It is well-known that b-quark does not interact significantly with NBS. Thus, we can add a b-quark (or anti-b-quark) to the NBS having eleven constituents without essential changing its energy, or mass. Then the b-replaced scalar NBS $T_{s}(b-$ replaced $)$, or $T_{s}(\bar{b}-$ replaced $)$, given by Eqs. (24) and (25) respectively, will have a mass $\simeq 300 \mathrm{GeV}$.

As to the NBS $T_{f}(b-$ replaced $)=5 t+b+5 \bar{t}$ and $T_{f}(b-$ replaced, $b \bar{b})=5 t+b+n_{b} b \bar{b}+5 \bar{t}$, they will have a mass very close to the NBS with ten constituents, e.g. $M_{T_{f}} \simeq 500 \mathrm{GeV}$ (see Fig. 3).

We also can consider more heavy T-balls with $M_{T}>500 \mathrm{GeV}$, but they will have very small cross-sections of their production.

The more accurate estimate given in Ref. [7] predicts the existence of "11" and "10" constituent bound states with masses approximately 760 and $960 \mathrm{GeV}$, respectively.

\section{New phases of the SM}

The existence of the new phases of the SM, different from the well-known Salam-Weinberg Higgs phase, leads to the confrontation with a question: Does a phase of the condensed $T_{s}$-balls exist?

The answer on this question is related with the SM parameters.

We can consider two phases I and II:

Phase-I does not have the Bose-Einstein condensate of $T_{s}$-balls. In this phase the VEV of the $T_{s}$-ball's scalar field $\Phi_{T}$ is equal to zero: $\left\langle\Phi_{T}\right\rangle=0$.

Phase-II contains such a condensate and $\left.<\Phi_{T}\right\rangle \neq 0$.

The main requirement of the appearance of the new phase of the condensed $T_{s}$-balls is a condition:

$$
m_{N B S}^{2}=M_{T_{s}}^{2}=0
$$

\subsection{Three EW phases of the SM}

Finally, taking into account seriously our results in the estimates of $g_{t}$ and $M_{T}$, we can consider three phases - three vacua of the SM at the EW-scale:

I) $<\Phi_{H}>\neq 0,<\Phi_{T}>=0$ — "Vacuum 1", the phase in which we live;

II) $<\Phi_{H}>\neq 0,<\Phi_{T}>\neq 0$ — Vacuum 2";

III) $<\Phi_{H}>=0,<\Phi_{T}>\neq 0$ - Vacuum 3",

which are presented symbolically by the phase diagram of Fig. 4. 


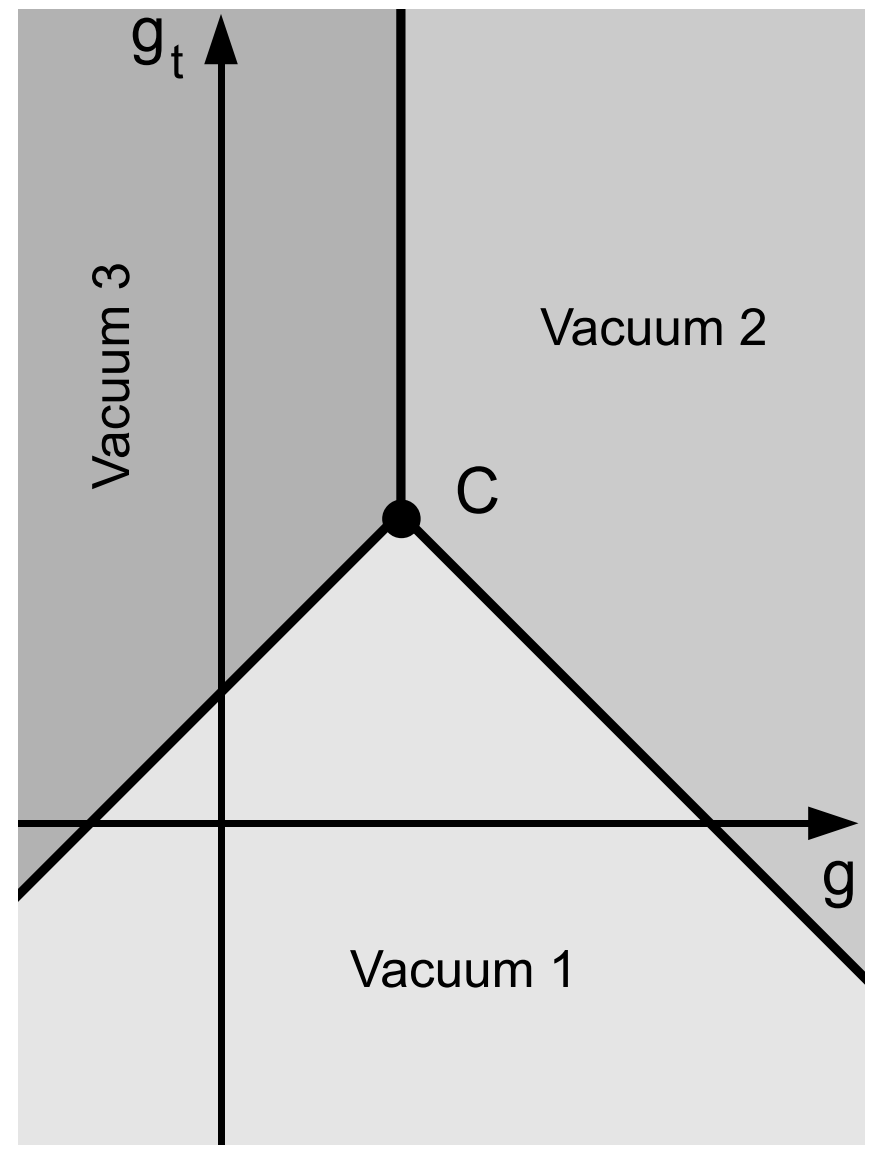

Fig. 4: A symbolic phase diagram for the SM at the EW-scale. 


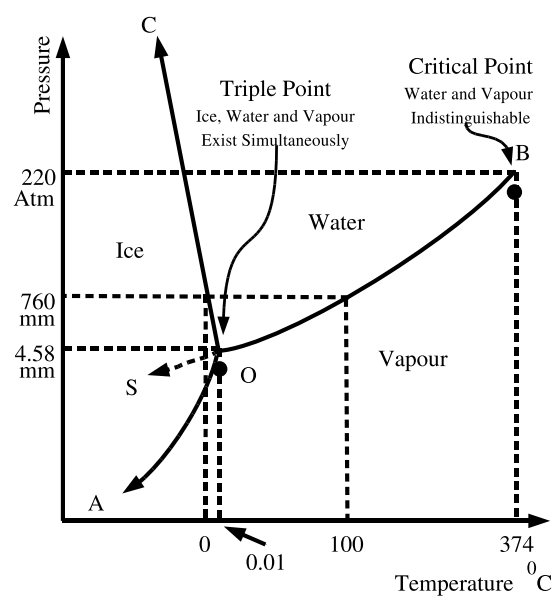

Fig. 5:

Fig. 4 shows the critical point $\mathrm{C}$ (triple point), in which the SM three phases meet together: this triple point is similar to the critical point considered in thermodynamics where the density of the vapor, water and ice are equal (see Fig. 5).

The existence of the new phases near the EW-scale can solve the problem of hierarchy. Here we recall the Multiple Point Principle (MPP) suggested in Refs. [33 40].

\subsection{The fundamental (Planck) scale of the SM}

A priori it is quite possible for a quantum field theory to have several minima of its effective potential as a function of its scalar fields $\Phi$ (exactly speaking of its norm $|\Phi|$ ). These minima can be degenerate. Moreover, it is assumed that all vacua existing in Nature (there can be a number of several vacua) are degenerate and have the same zero, or almost zero, vacuum energy densities which coincide with the cosmological constant $\Lambda$ determined by Einstein. This is confirmed by the phenomenological cosmology.

According to the MPP, the SM has the two minima of its effective potential considered as a function of the variable $|\Phi|$, where $\Phi=\Phi_{H}$. These minima are degenerate and have $\Lambda=0$ :

$$
\begin{aligned}
\left.V_{\mathrm{eff}}\right|_{\min 1} & =\left.V_{\mathrm{eff}}\right|_{\min 2}=0, \\
\left.\mathbf{V}_{\mathrm{eff}}^{\prime}\right|_{\min 1} & =\left.\mathbf{V}_{\mathrm{eff}}^{\prime}\right|_{\min \mathbf{2}}=\mathbf{0},
\end{aligned}
$$

what is shown in Fig. 6.

It is assumed that the second minimum exists near the Planck scale:

$$
\left|\Phi_{\min 2}\right| \sim M_{\mathrm{Pl}}
$$

This is a fundamental scale. 


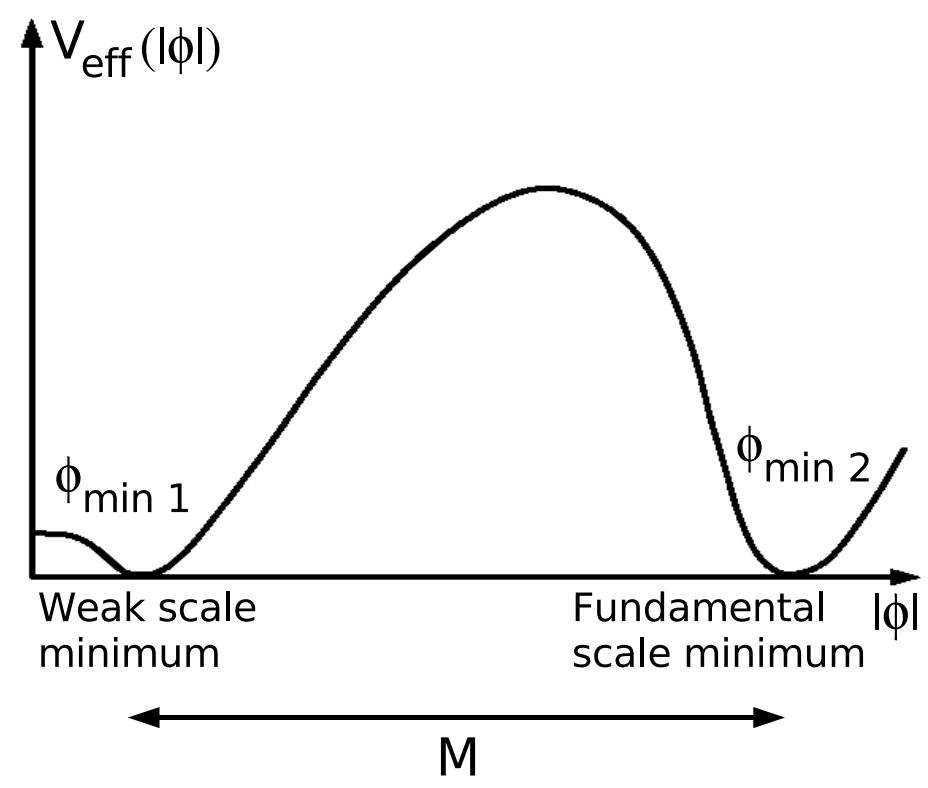

Fig. 6: The first (our) vacuum at $|\Phi| \approx 246 \mathrm{GeV}$ and the second vacuum at the fundamental scale $|\Phi| \sim M_{\mathrm{Pl}}$.

The calculation of the NBS masses is based only on the SM parameters. The MPP determines the coupling constants in the SM and therefore - the structure of the NBSs $T_{s, f}$. Since at the border of the two phases I and II the top-quark Yukawa coupling constant leads to zero mass of the NBS $T_{s}$, we can assume that the MPP manifests the phase transitions in the SM in such a way that we have the finetuning in the SM, which solves the hierarchy problem. The MPP calculations of gauge coupling constants were obtained in Refs. [41, 42,

\section{Can we observe T-balls at LHC or Tevatron?}

If the mean square radius of the T-ball is small in comparison with its Compton wave length:

$$
r_{0} \approx\left(\sqrt{2} M_{t}\right)^{-1}<<\frac{1}{m_{N B S}},
$$

then the NBS can be considered as an almost fundamental particle.

The fermionic NBS $T_{f}$ is similar to the $t^{\prime}$-quark of the fourth generation belonging to the fundamental representation $\underline{3}$ (color triplet).

Then our NBS are strongly bound and can be observed at colliders (Tevatron, LHC, etc.) in the following processes: 
1) First of all, in the possible H-decay process:

$$
H \rightarrow 2 T_{s},
$$

if $M_{T_{s}}<\frac{1}{2} M_{H}$. Using limits given by Tevatron experiments [2]: $114 \lesssim M_{H} \lesssim 158 \mathrm{GeV}$, we obtain the requirement for the Higgs decay mechanism:

$$
M_{T_{s}} \lesssim 80 \mathrm{GeV} \text {. }
$$

Here we have argued that T-balls can explain why it is difficult to observe the Higgs boson $\mathrm{H}$ at colliders: T-balls can strongly enlarge the decay width of the Higgs particle.

2) If $M_{T_{s}}>\frac{1}{2} M_{H}$, then the first decay (35) is absent in Nature, and the $f T_{s}$-balls fly away, forming jets which produce hadrons with a high multiplicity:

$$
T_{s} \rightarrow J E T S \text {. }
$$

3) Second, we can observe at Tevatron all processes given by Fig. 7 with the replacement $t \bar{t} \rightarrow t^{\prime} \bar{t}^{\prime}, T_{f} \overline{T_{f}}$. In the most optimistic cases the NBS $6 t+5 \bar{t}$ (fermionic fireball) plays a role of the fundamental quark of the fourth generation, say, with the mass $M_{T_{f}} \gtrsim 300$ $\mathrm{GeV}$, given by our preliminary estimate. We expect that the Tevatron-LHC experiments should find either a fourth family t'-quark, or the fermionic NBS $T_{f}$, or both of them.

The scalar NBS $T_{s}$ cannot be produced simply in a pair by a gluon vertex, because it is a color singlet $\underline{\mathbf{1}}$. But a pair $T_{f} \overline{T_{f}}$ can be produced by a gluon, because $T_{f}$ is a color triplet $\underline{\mathbf{3}}$.

At LHC the pairs of $T_{s}$-balls, or $T_{f}$-balls might be produced in $p p$ collisions via the two gluon diagram with strong vertices shown in Fig. 8 [3,43].

\section{CDF II Detector experiment at the Tevatron}

Recent experiments with CDF II Detector of the Tevatron [1] searching for heavy top-like quarks in $p \bar{p}$-collisions with $\sqrt{s} \simeq 1.96 \mathrm{TeV}$ do not exclude the existence of T-balls with masses $\gtrsim 300 \mathrm{GeV}$ up to $500 \mathrm{GeV}$.

Here we can assume that the very strange events observed at the Tevatron as a fourth family $t^{\prime}$, which decays into a $W$-boson and a presumed quark-jet, might find another explanation in our model: maybe it is a decay of T-balls into a $W$-boson and a gluon jet.

Tevatron experiments exclude a fourth-generation t' quark with a mass below 300 $\mathrm{GeV}$ (see Refs. [1]). Assuming that fourth generation $t^{\prime}$-quarks does not exist in Nature, but only the pairs of fermionic NBS $T_{f}$ are produced at the Tevatron, we can give an explanation of the observed cross-sections shown in Fig. 9.

The curve for the cross-section

$$
\sigma\left(p \bar{p} \rightarrow t^{\prime} \bar{t}^{\prime}\right) \simeq 0.1 p b
$$

can correspond to the production of pairs of fermionic $T_{f}$-balls with mass $M_{T_{f}} \gtrsim 300$ $\mathrm{GeV}$. 


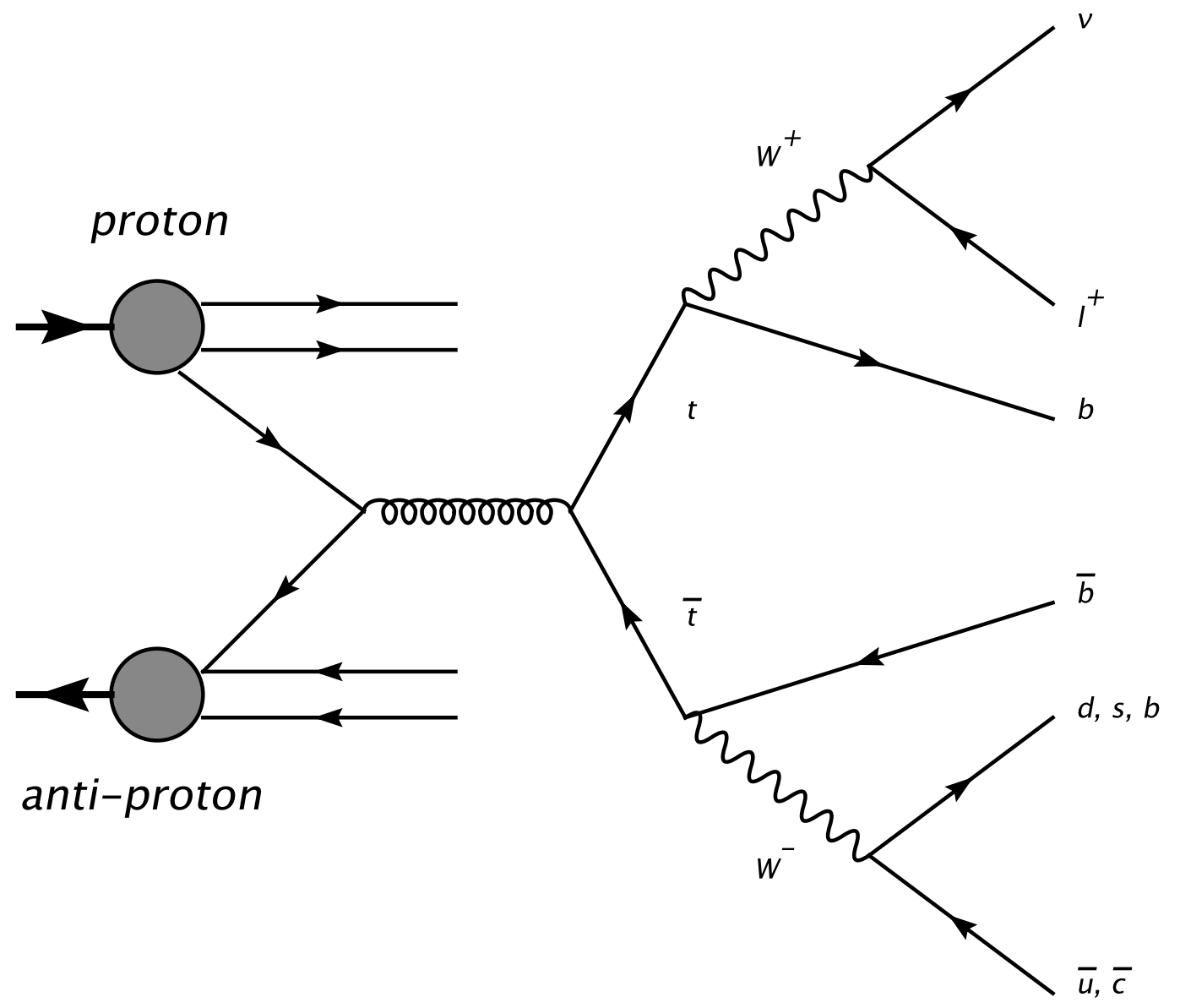

Fig. 7: A typical process observed at the Tevatron in $p \bar{p}$ collisions. 


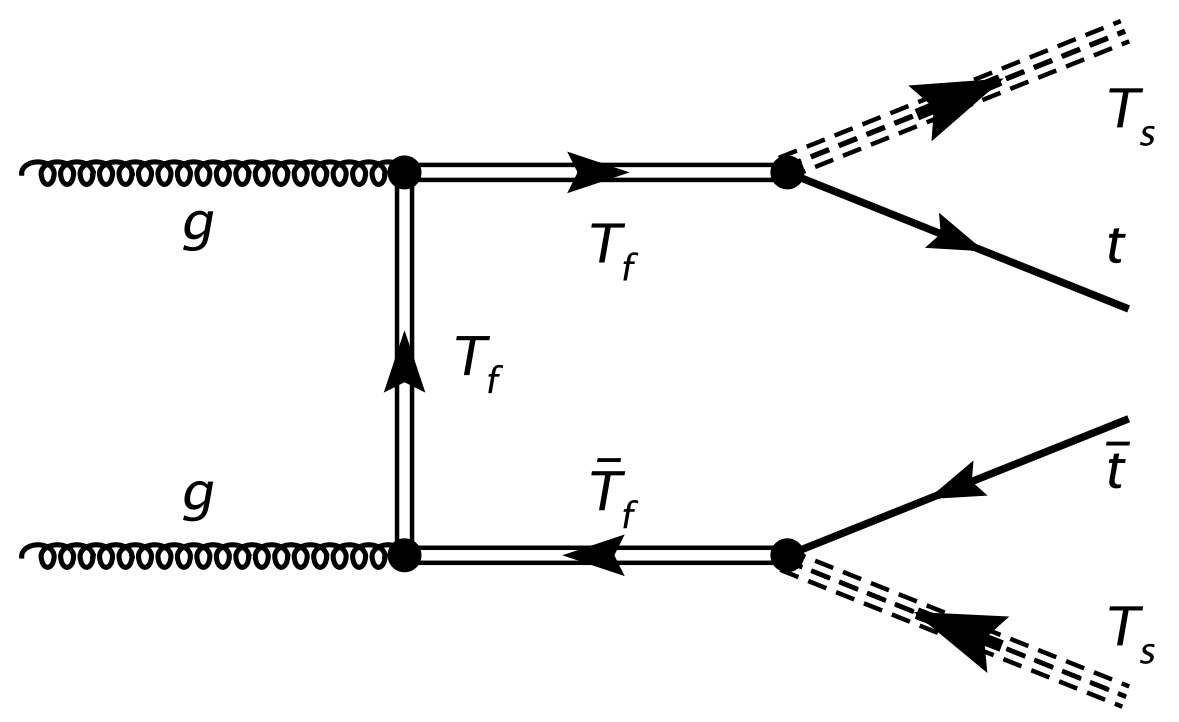

Fig. 8: Two gluon production of $T_{s}$-balls

\section{Estimate of the NBS form-factors in the Tevatron CDF-experiment}

Assuming that only the fermionic $T_{f}$-balls with mass $M_{T}>300 \mathrm{GeV}$ are produced at the Tevatron in the CDF-experiment [1], we can imagine the existence of form-factors of the NBS $T_{f}$, which determine the cross-section of the production of the fermionic T-balls (see Fig. 9):

$$
\sigma\left(p \bar{p} \rightarrow T_{f} \overline{T_{f}}\right)=F^{2}\left(M_{T}\right) \sigma_{\text {theor }}\left(M_{T}\right)
$$

Here $\sigma\left(p \bar{p} \rightarrow T_{f} \overline{T_{f}}\right)$ is given by the observed red line curve of Fig. 9 and $\sigma_{\text {theor }}\left(M_{T}\right)$ is given by the theoretical (blue) curve obtained by Bonciani et al. 44,45] for the point-like particle $t^{\prime}$. Our numerical calculations of the form-factor shown in Fig. 10 gives the results in the region of $M_{T}$ from $311 \mathrm{GeV}$ (where $F\left(M_{T}\right)=1$ ) up to $500 \mathrm{GeV}$. We conclude that for $M_{T}=500 \mathrm{GeV}$ the form-factor is large enough:

$$
F\left(M_{T}\right) \approx 7.6 .
$$

\section{Charge multiplicity in decays of T-balls}

Actually Li and Nielsen suggested in Ref. [43] that the NBSs would decay to a rather low number of jets, but at first one might very reasonably think that since we have to do with bound states of very many constituents and actually $6 t \bar{t}$ pairs, it sounds that the possibility of them decaying into as many jets as there are pairs to annihilate, say - or 


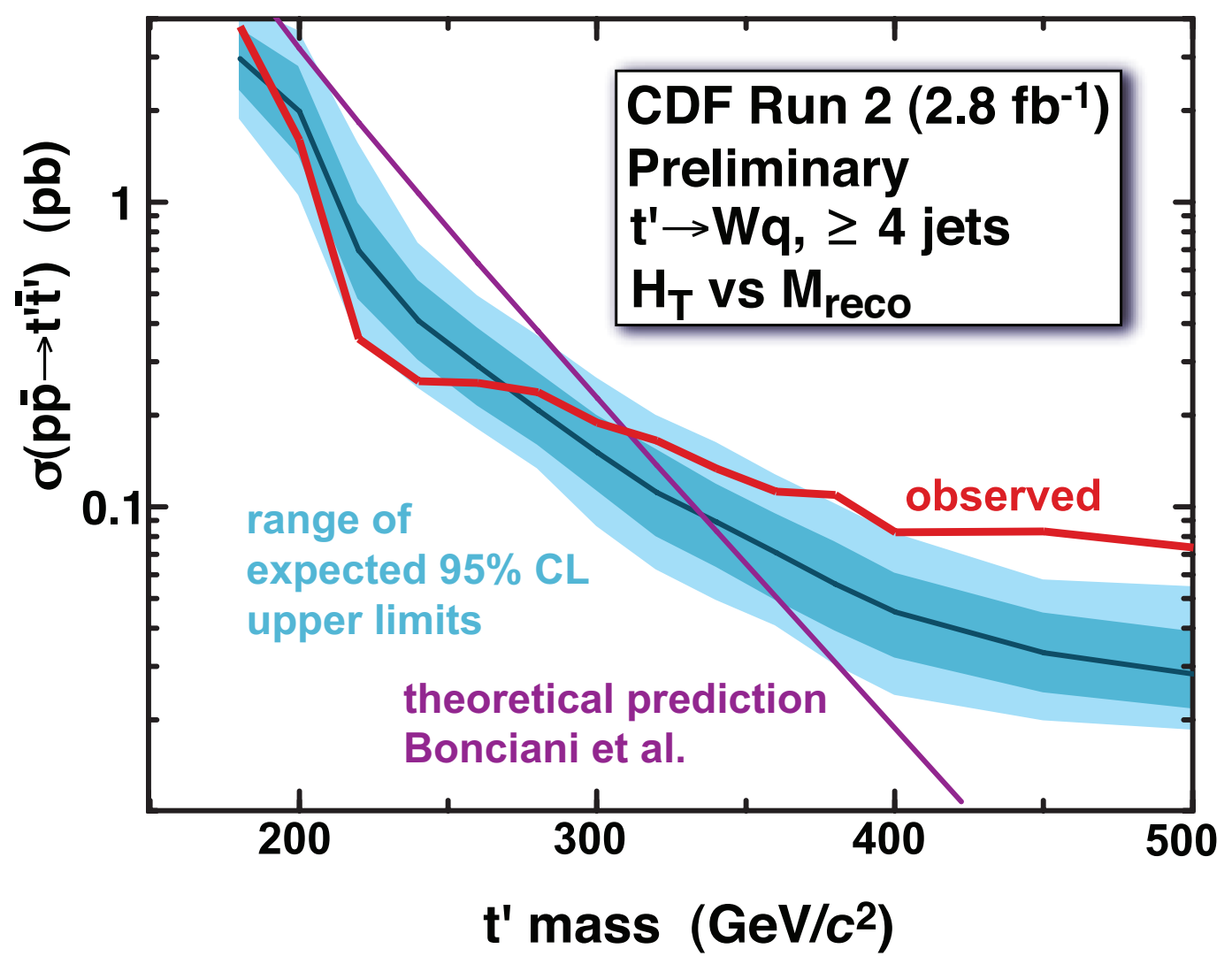

Fig. 9: Tevatron CDF-experiment given by Refs. [1]: upper limit, at 95\% CL, a fourthgeneration t' quark with a mass below $300 \mathrm{GeV}$ is excluded. Blue line presents a theoretical curve for the fourth-generation quarks cross-section. 


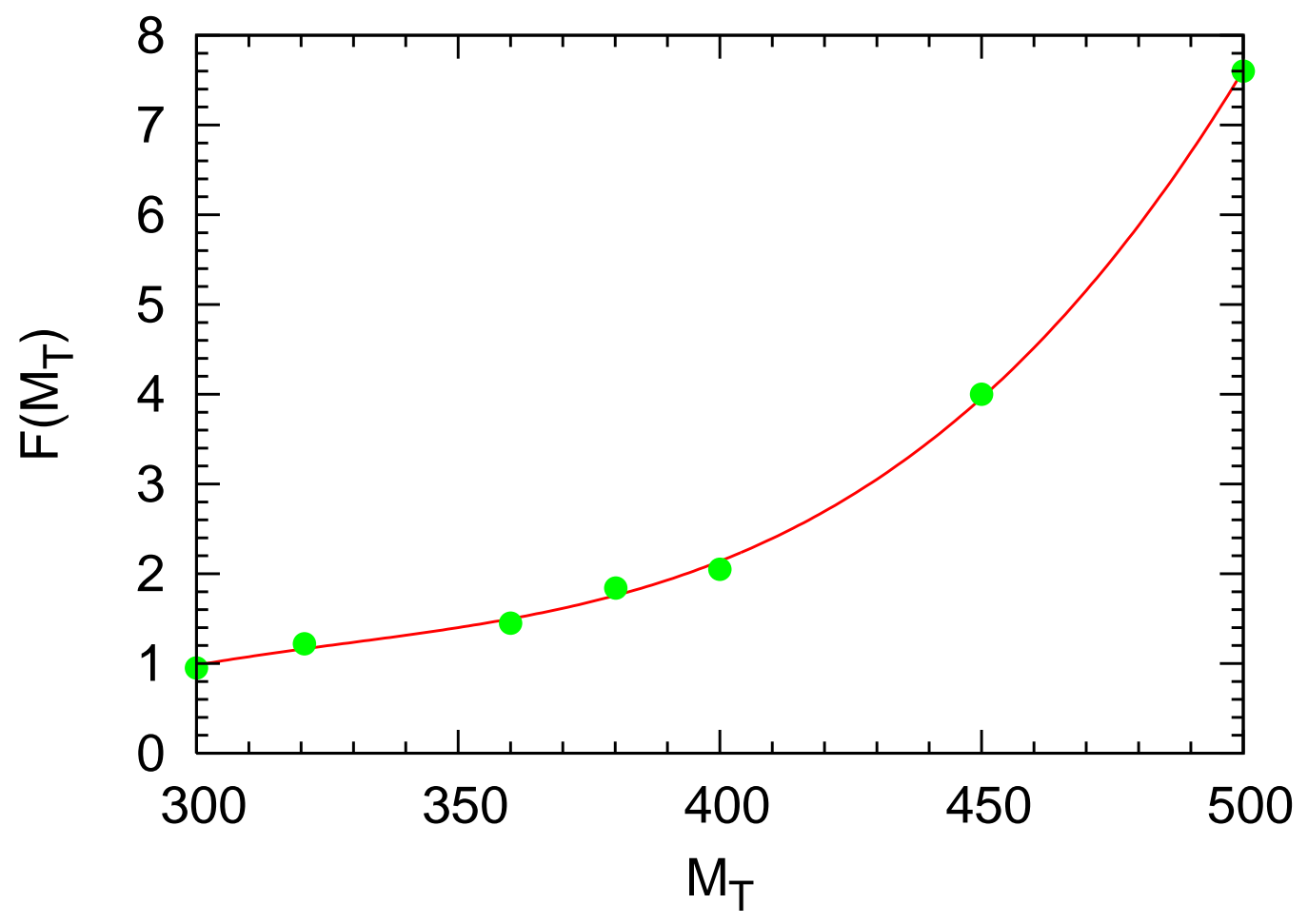

Fig. 10: The form-factor $F\left(M_{T}\right)$ of the fermionic new bound state $T_{f}$ obtained from Tevatron CDF-experiment [1] in absence of the four generation. 
even the number of constituents - has some intuitive appeal and should not just be thrown away as a possibility by the Li-Nielsen rather non-safe argument. We shall therefore here develop what we would expect in the case of the separate $t \bar{t}$ pairs decaying essentially separately, although we do not really believe that any longer: if the mass of the NBS, containing 6 pairs of $t \bar{t}$, is $M_{S}$, then the energy per one annihilation of $t \bar{t}$ approximately is equal to the following value:

$$
E_{\text {an }}=E_{(\text {for one annihilation })} \approx \frac{1}{6} M_{S}
$$

e.g.

$$
E_{(\text {for one annihilation })} \approx 10 \mathrm{GeV},
$$

if

$$
M_{S} \approx 60 \mathrm{GeV} \text {. }
$$

In this case, during the annihilation produced by $e^{+} e^{-}$-collisions, the special charge multiplicity is

$$
<N_{c h}\left(e^{+} e^{-}\right)>\approx 10
$$

while the annihilation produced by $p p$-collisions, the special charge multiplicity is

$$
<N_{c h}(p p)>\approx 6 .
$$

Such calculations of $\left\langle N_{c h}>\right.$ vs $E_{a n}$ are based on the investigation of Ref. [46]. Here for $M_{S} \approx 60 \mathrm{GeV}$ we obtain the following values for the charge multiplicity:

$$
\begin{gathered}
N_{c h}\left(e^{+} e^{-}\right) \approx 6 \cdot 10 \approx 60, \\
N_{c h}(p p) \approx 6 \cdot 6 \approx 36 .
\end{gathered}
$$

The value of the charge multiplicity weakly depends on the NBS mass. For instance, if $M_{S} \approx 80 \mathrm{GeV}$, then:

$$
<N_{c h}(p p)>\approx 6.5
$$

and

$$
N_{c h}(p p) \approx 6 \cdot 6.5 \approx 39
$$

But if $M_{S} \approx 100 \mathrm{GeV}$, then:

$$
<N_{c h}(p p)>\approx 7
$$

and

$$
N_{c h}(p p) \approx 6 \cdot 7 \approx 42
$$

However, such a maximally possible charge multiplicity will not be realized in practice, because between the produced in the final state pairs $t \bar{t}$, or $b \bar{b}$, can exist extra exchanges by gluons and the Higgs bosons giving new annihilations. And we shall obtain less jets. 


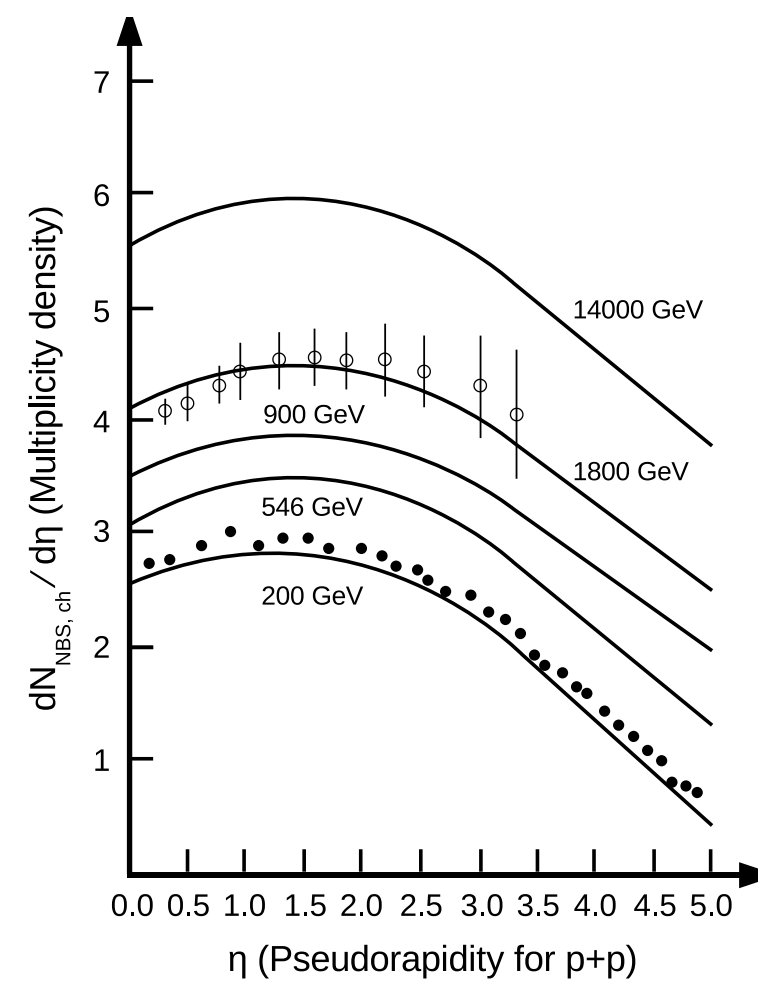

Fig. 11:

Indeed, it would be very strange if the decay width of the T-balls was small. Then we would have narrow peaks in JETS. It would be exactly a good way to see that our model were right if you could find some narrow peak in the distribution of the total mass of some JETS.

For $p p$-collisions the estimates [43] give :

$$
\left.\frac{d N_{c h}}{d \eta}\right|_{\max } \approx 6 .
$$

Such a value is expected for this derivative at LHC (see Fig. 11). The maximum of this curve corresponds to the LHC energy $W=14 \mathrm{TeV}$ in $p p$-collisions. 


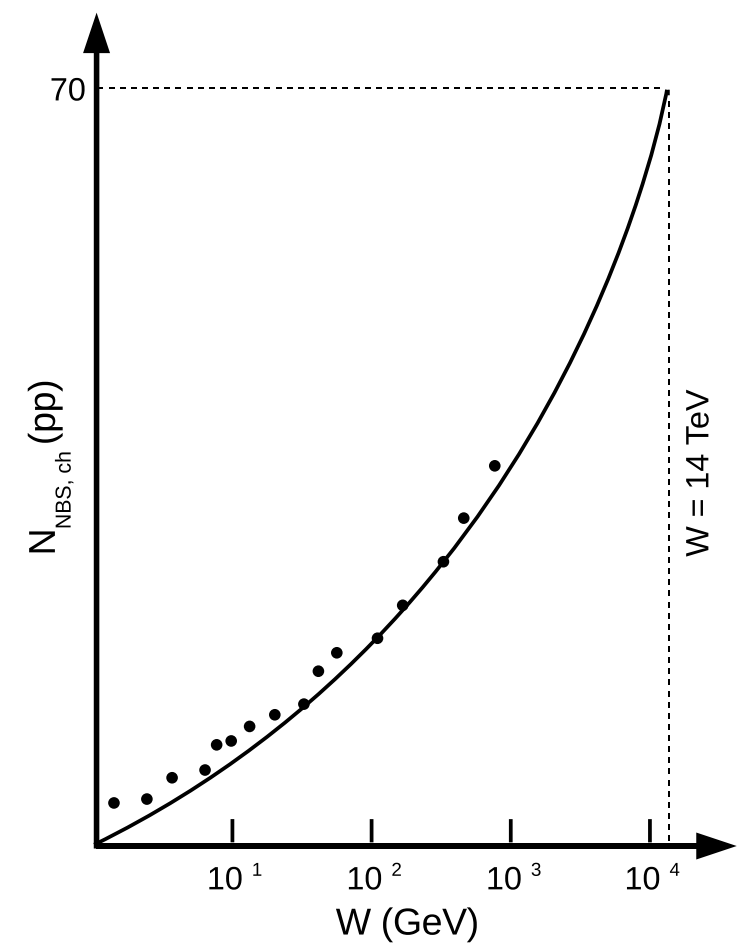

Fig. 12:

The dependence $N_{\mathrm{ch}}$ vs $W$ is presented in Fig. 12. Here

$$
\left.N_{c h}(p p)\right|_{W=14 T e V} \approx 65 \text {. }
$$

These calculations (figures) show that T-balls can give an essential contributions to charge multiplicity in $p p$-collisions, provided that their decays really go as if each $t \bar{t}$ pair decayed separately and not as the recent estimate by Li and Nielsen [43].

\section{Conclusions}

At present, a lot of physicists, theorists and experimentalists, are looking forward to the New Physics. However, it is quite possible that LHC will discover only the SalamWeinberg Higgs boson and nothing more. Nevertheless, the T-balls considered in the present paper could exist in the framework of the SM.

1. The present investigation devoted to the main problems of the Standard Model is based on the following three assumptions: 1) there exists $1 S$-bound state of $6 t+6 \bar{t}$, e.g. bound state of 6 quarks of the third generation with their 6 anti-quarks; 2) the forces 
which bind these top-quarks are so strong that they almost completely compensate the mass of the 12 top-quarks forming this bound state; 3) such strong forces are produced by the Higgs interactions: the interactions of top-quarks via the virtual exchange of the scalar Higgs bosons coupling with a large value of the top-quark Yukawa coupling constant.

A new bound state $6 t+6 \bar{t}$, which is a color singlet, was first suggested by Froggatt and Nielsen and now is named 'T-ball'.

2. Present theory also predicts the existence of a new bound state $6 t+5 \bar{t}$, which is a color triplet and a fermion similar to the quark of the fourth generation.

3. We have also considered "b-replaced" NBSs: $T_{S}\left(n_{b} b-\right.$ replaced $)=n_{b} b+(6 t+$ $\left.6 \bar{t}-n_{b} t\right)$ and $T_{f}\left(n_{b} b-\right.$ replaced $)=n_{b} b+\left(6 t+5 \bar{t}-n_{b} t\right)$, where $n_{b}$ is the integer number. The presence of b-quarks in the NBS leads to the dominance of the isospin singlets: with the inclusion of both $b$ and $t$ quarks we obtain a more weak isospin invariant picture.

4. We have estimated the masses of the lightest "b-replaced" NBSs: $M_{T(b-\text { replaced })} \simeq$ $(300-400) \mathrm{GeV}$, and predicted the existence of the more heavy "b-replaced" NBSs: $M_{T\left(n_{b} b-\text { replaced }\right)}>400 \mathrm{GeV}$ with $n_{b}>1$.

5. We have developed a theory of T-ball's condensate, and predicted the possibility of the existence of three SM phases at the EW-scale. Calculating the top-quark Yukawa coupling constant at the border of two phases (with T-ball's condensate and without it) we have obtained $g_{t} \approx 1$.

6. It was shown that CDF II Detector experiment searching for heavy top-like quarks at the Tevatron (in $p \bar{p}$-collisions with $\sqrt{s} \simeq 1.96 \mathrm{GeV}$ ) can observe $T_{f}$-balls with masses up to $400 \mathrm{GeV}$.

7. We have considered all processes with T-balls, which can be observed at LHC, especially the decay

$$
H \rightarrow 2 T_{s}
$$

and the production of $T_{f} \overline{T_{f}}$ as an alternative of the $t^{\prime} \overline{t^{\prime}}$ production (where $t^{\prime}$ is the quark of the fourth generation with t-quark quantum numbers).

8. We have constructed the possible form-factors of T-balls.

9. We have estimated the charge multiplicity (coming from the T-ball's decays) at the energy $\mathrm{W}=14 \mathrm{TeV}$ at LHC.

\section{Acknowledgments}

We deeply thank for the courtesy of CDF collaboration for the presentation of figures from there.

H.B.N. is grateful to J. Conway, R. Erbacher, J. Frost, H. Jensen, C. Issever, E. Lytken, K. Loureiro and A. Parker for advices and fruitful discussions.

L.V.L. thanks A.B. Kaidalov, O.V. Pavlovsky and M.A. Trusov for useful discussions. 


\section{References}

[1] 1. CDF Collaboration (T. Aaltonen et al.). FERMILAB-PUB-11-015-E, Jan 2011; arXiv:1101.4926 [hep-ex].

2. CDF Collaboration (T. Aaltonen et al.). FERMILAB-PUB-10-525-E, Jan 2011: arXiv:1101.0034 [hep-ex].

3. CDF Collaboration and D0 Collaboration (D. Benjamin for the collaboration) in: Proceedings of 44th Rencontres de Moriond EW 2009: Electroweak Interactions and Unified Theories, La Thuile, Italy, 7-14 March 2009, arXiv: 0906.1403 [hep-ex].

4. CDF Collaboration and D0 Collaboration (S. Pagan Griso for the collaboration) in: Proceedings of 44th Rencontres de Moriond on QCD and High Energy Interactions, La Thuile, Valle d'Aosta, Italy, 14-21 March 2009, arXiv: 0905.2090 [hep-ex].

[2] OPAL Collaboration (G. Abbiendi et al.), Search for a low mass CP odd Higgs boson in e+ $e$ - collisions with the OPAL detector at LEP-2, Eur.Phys.J.C 27, 483 (2003) [arXiv: hep-ex/0209068].

[3] C.D. Froggatt, L.V. Laperashvili, R.B. Nevzorov and H.B. Nielsen, The Production of $6 t+6 \bar{t}$ bound state at colliders. A talk given by Holger Bech Nielsen at CERN, 2008, preprint CERN-PH-TH/2008-051.

[4] C.R. Das, C.D. Froggatt, L.V. Laperashvili, H.B. Nielsen, New Bound States of Top and Beauty Quarks at the Tevatron and LHC. Talk given by L.V. Laperashvili at 14th Lomonosov Conference on Elementary Particle Physics, Moscow, Russia, 19-25 Aug 2009. Published in Particle Physics at the Year of Astronomy, in: Proceedings of the Fourteenth Lomonosov Conference on Elementary Particle Physics, 379 - 381, Editor: Alexander I. Studenikin, World Scientific Publishing Company,2010 e-Print: arXiv:0908.4514 [hep-ph]

[5] C.D. Froggatt and H.B. Nielsen, Trying to understand the Standard Model parameters Invited talk by H.B. Nielsen at the "XXXI ITEP Winter School of Physics, Moscow, Russia, 18-26 February 2003, Surveys High Energy Phys 18, 55-75 (2003) [arXiv: hep-ph/0308144.

[6] C.D. Froggatt, H.B. Nielsen and L.V. Laperashvili, Hierarchy-problem and a bound state of $6 t$ and 6 anti-t in: Proceedings of Coral Gables Conference on Launching of Belle Epoque in High-Energy Physics and Cosmology (CG 2003), Ft. Lauderdale, Florida, 17-21 December 2003, published in Int.J.Mod.Phys.A 20, 1268 (2005) [arXiv: hep-ph/0406110].

[7] C.D. Froggatt and H.B. Nielsen, Phys.Rev.D 80,034033 (2009); arXiv:0811.2089 [hep-ph]. 
[8] C.D. Froggatt and H.B. Nielsen, New Bound States of several Top-quarks bound by Higgs Exchange in: Proceedings of the 34th Int.Conf. on High Energy Physics, Philadelfia, 2008, arXiv:0810.0475 [hep-ph].

[9] C.D. Froggatt, L.V. Laperashvili and H.B. Nielsen, A New bound state $6 t+6$ anti-t and the fundamental-weak scale hierarchy in the Standard Model in: Proceedings of 13th International Seminar on High-Energy Physics: QUARKS-2004, Pushkinskie Gory, Russia, 24-30 May 2004 [arXiv: hep-ph/0410243].

[10] C.D. Froggatt, The Hierarchy problem and an exotic bound state in: Proceedings of 10th International Symposium on Particles, Strings and Cosmology, (PASCOS 04 and Pran Nath Fest), Boston, Massachusetts, 16-22 Aug 2004. Published in: "Boston 2004, Particles, strings and cosmology", pp.325-334 [arXiv: hep-ph/0412337].

[11] C.D. Froggatt, L.V. Laperashvili and H.B. Nielsen, The Fundamental-weak scale hierarchy in the Standard Model, Phys.Atom.Nucl. 69, 67 (2006) [Yad.Fiz. 69, 3 (2006), [arXiv: hep-ph/0407102].

[12] C.D. Froggatt, L.V. Laperashvili, R.B. Nevzorov, H.B. Nielsen and C.R. Das, New Bound States of Top-anti-Top Quarks and T-balls Production at Colliders (Tevatron, LHC, etc.), arXiv: 0804.4506 [hep-ph].

[13] M.Yu. Kuchiev, Phys.Rev.D 82, 127701 (2010), arXiv:1009.2012 [hep-ph].

[14] M.Yu. Kuchiev, V.V. Flambaum and E. Shuryak, Phys.Rev. D78, 077502 (2008) [arXiv: 0808.3632 [hep-ph]], arXiv: 0811.1387.

[15] M.Yu. Kuchiev, V.V. Flambaum and E. Shuryak, Phys.Lett.B 693, 485 (2010), arXiv:0811.1387 [hep-ph].

[16] Jean-Marc Richard, About the stability of the dodecatoplet, Few Body Syst. 45, 65 (2009) [arXiv: 0811.2711 [hep-ph]].

[17] Yu.P. Goncharov, Nucl.Phys. A808, 73 (2008) [arXiv: 0806.4747 [hep-ph]].

[18] Y. Kiyo and Y. Sumino, Phys.Rev. D67, 071501 (2003) [arXiv: hep-ph/0211299].

[19] W. Kummer and W. Modritsch, Nucl.Phys. B430, 3 (1994) [ArXiv: hep-ph/9307202]; Phys.Lett. B349, 525 (1995) [arXiv: hep-ph/9501406].

[20] N. Fabiano, A. Grau and G. Pancheri, Phys.Rev. D50, 3173 (1994); Nuovo Cim. A 107, 2789 (1994).

[21] J.H. Kuhn and E. Mirkes, Phys.Rev. D48, 179 (1993) [arXiv: hep-ph/9301204]. 
[22] A.L. Macpherson and B.A. Campbell, Phys.Lett. B306, 379 (1993) [arXiv: hep-ph/9302278.

[23] Particle Data Group, C. Amster et al., Phys.Lett. B667, 1 (2008).

[24] A. Chodos, R.L. Jaffe, K. Johnson, C.B. Thorn and V.F. Weisskopf, Phys.Rev. D9, 3471 (1974).

[25] W.A. Bardeen, M.S. Chanowitz, S.D. Drell, M. Weinstein and T.-M. Yan, Phys.Rev. D11, 1094 (1975).

[26] R. Friedberg and T.D. Lee, Phys.Rev. D15, 1694 (1977).

[27] R. MacKenzie, F. Wilczek and A. Zee, Phys.Rev.Lett. 53, 2203 (1984).

[28] R. MacKenzie, Mod.Phys.Lett. A7, 293 (1992).

[29] Y. Nambu and G. Jona-Lasinio, Phys.Rev. 122, 345 (1961).

[30] W.A. Bardeen, C.T.Hill and M. Lindner, Phys.Rev. D41, 1647 (1990).

[31] V.A. Miransky, M. Tanabashi and K. Yamawaki, Phys.Lett. B221, 177 (1989); Mod.Phys.Lett. A4, 1043 (1989).

[32] G. Cvetic, Rev.Mod.Phys. 71, 513 (1999) [arXiv: hep-ph/9702381].

[33] D.L. Bennett, H.B. Nielsen, Int.J.Mod.Phys. A 9, 5155 (1994); ibid., A 14, 3313 (1999).

[34] D.L.Bennett, C.D.Froggatt, H.B.Nielsen, in Proceedings of the 27th International Conference on High Energy Physics, Glasgow, Scotland, 1994, Ed. by P.Bussey and I.Knowles (IOP Publishing Ltd, 1995), p.557; Perspectives in Particle Physics '94, Ed. by D.Klabučar, I.Picek and D.Tadić (World Scientific, Singapore, 1995), p.255; arXiv: hep-ph/9504294;

[35] C.D.Froggatt and H.B.Nielsen, Origin of Symmetries (World Sci., Singapore, 1991).

[36] L.V.Laperashvili, Yad.Fiz. 57, 501 (1994) [Phys.At.Nucl. 57, 471 (1994)].

[37] C.D.Froggatt, H.B.Nielsen, Phys.Lett. B368 (1996) 96.

[38] C.D. Froggatt, L.V. Laperashvili, R.B. Nevzorov and H.B. Nielsen, Phys.Atom.Nucl. 67, 582 (2004) [Yad.Fiz. 67, 601 (2004)]; arXiv: hep-ph/0310127.

[39] L.V. Laperashvili. The Multiple point principle and Higgs bosons. Phys.Part.Nucl. 36 S38-S40 (2005), arXiv: hep-ph/0411177. 
[40] C.R. Das, L.V. Laperashvili, Int.J.Mod.Phys. A 20, 5911 (2005).

[41] D.L. Bennett, L.V. Laperashvili, H.B. Nielsen, Relation between fine structure constants at the Planck scale from multiple point principle, in: Proceedings to the 9th Workshop: What comes beyond the standard models, Bled, Slovenia (DMFA, Zaloznistvo, Ljubljana, M. Breskvar et al., Dec 2006), p.10; arXiv: hep-ph/0612250.

[42] D.L. Bennett, L.V. Laperashvili, H.B. Nielsen, Finestructure constants at the Planck scale from multiple point principle, in: Proceedings to the 10th Workshop on What Comes Beyond the Standard Model, Bled, Slovenia, 17-27 Jul 2007; arXiv: 0711.4681 [hep-ph].

[43] S.Y. Li and H.B. Nielsen. Jet Multiplicity for Hard Bound State, and Mass Calculations for Bound States of Several Top- and Anti-Top Quarks, CERN-preprints, to be published.

[44] R. Bonciani, A. Ferroglia, T. Gehrmann, D. Maitre and C. Studerus, JHEP 0807, 129 (2008) [arXiv: 0806.2301]; arXiv: 0810.0598.

[45] R. Bonciani, S. Catani, M.L. Mangano and P. Nason, Nucl.Phys.B 529, 424 (1998), Erratum-ibid. B 803, 234 (2008) [arXiv: hep-ph/9801375].

[46] E.K.G. Sarkisyan, A.S. Sakharov. Multihadron production features in different reactions. Invited talk at 35th International Symposium on Multiparticle Dynamics (ISMD 05), Kromeriz, Czech Republic, 9-15 Aug 2005. Published in: AIP Conf.Proc. 828, 35-41 (2006). Also in: *Kromeriz 2005, Multiparticle dynamics*, 35-41; arXiv: hep-ph/0510191. 